\title{
Characterization of the Grain Size in Ferromagnetic Colloids: Comparing Torsional-Pendulum Measurements with Standard Complementary Methods
}

By J. Embs ${ }^{1,2}$, H. W. Müller ${ }^{4}$, C. E. Krill III ${ }^{2}$, F. Meyer ${ }^{3}$, H. Natter ${ }^{3}$,

B. Müller ${ }^{4}$, S. Wiegand ${ }^{4}$, M. Lücke ${ }^{1}$, K. Knorr ${ }^{2}$, and R. Hempelmann ${ }^{3, *}$

1 Theoretische Physik, Universität des Saarlandes, Postfach 151150,

D-66041 Saarbrücken, Germany

2 Technische Physik, Universität des Saarlandes, Postfach 151150,

D-66041 Saarbrücken, Germany

${ }^{3}$ Physikalische Chemie, Universität des Saarlandes, Postfach 151150, D-66041 Saarbrücken, Germany

${ }^{4}$ Max-Planck-Institut für Polymerforschung, Ackermannweg 10, D-55128 Mainz, Germany

(Received September 27, 2005; accepted in revised form November 16, 2005)

Ferrofluids / Particle Size Analysis / Relaxation Time

A recently introduced shear-flow-free method for measuring the rotational viscosity of a resonantly forced torsional pendulum is used to determine the transverse magnetic relaxation time in magnetite and cobalt-based ferrofluids. From these data the average size of the ferromagnetic grains and their hydrodynamic diameter (core plus surfactant coating) are deduced under in-situ conditions, i.e. without diluting the sample. The reliability of the method is demonstrated by comparing the results with those of the complementary techniques of magneto-granulometry, X-ray diffraction, electron microscopy, and photoncorrelation spectroscopy.

\section{Introduction}

Ferrofluids are liquid suspensions of monodomain ferromagnetic particles having a typical core diameter of $D \simeq 10 \mathrm{~nm}$ [1]. Each particle carries a permanent magnetic moment, which is proportional to the volume of the ferromagnetic core. To suppress the particle agglomeration that would result from dipoledipole or Van der Waals attractions, the particles are electrostatically stabilized

\footnotetext{
* Corresponding author. E-mail: r.hempelmann@mx.uni-saarland.de
} 
or covered with a surfactant coating, which prevents them from approaching each other too closely. The hydrodynamic diameter $D^{\text {hyd }}$ of the resulting ferrofluid particles (core plus surfactant coating) has a significant influence on the interaction between the particles and the carrier liquid. Many phenomena in ferrofluids, such as the viscosity enhancement in a static magnetic field [2,3], "negative viscosity" [4-6], and magneto-vortical resonance [7], are direct results of this interaction.

It is now possible to determine the core-size distribution $g(D)$ of ferromagnetic colloids using standard methods. For a magnetite-based ferrofluid suspended in a synthetic ester, a comparative investigation [8] revealed that the size distributions obtained by the methods of small-angle scattering, wideangle X-ray diffraction and transmission electron microscopy are consistent with each other, provided systematic uncertainties and differences in method are properly taken into account. For the determination of the hydrodynamic particle diameter, dynamic light scattering is a convenient tool, as long as the sample in question is transparent. Since ferrofluids of technically relevant concentrations (volume fraction $\varphi$ of a few percent) are strongly absorbent, they must be highly diluted in order to avoid the multiple scattering that renders light-scattering measurements inaccurate. The same is true of transient birefringence measurements, which have also been undertaken to determine the hydrodynamic diameter in ferrofluids [9]. Regardless of the type of particle coating (electrostatic or polymeric), the hydrodynamic size deduced by the latter method is always much larger than the core diameter, with ratios $D^{\text {hyd }} / D$ generally falling between 2 and 10 . For typical ferrofluids with a volume fraction of $\varphi \simeq 3 \%$, these values are unphysically high, because the hydrodynamic shells of the particles at those concentrations begin to overlap when $D^{\text {hyd }}>2.5 D$. The measurement of unrealistically high diameter ratios has been attributed to modifications in the microstructure of the ferrofluid particles caused by the dilution process. For example, the surfactant layer coating the ferromagnetic cores may become swollen (e.g. by bound water) under the influence of the dilution agent. Moreover, the addition of the dissolving agent disturbs the concentration equilibrium between the tenside molecules in solution and those adsorbed on the particle surfaces; consequently, the quality of the surfactant layer can deteriorate and the particles begin to agglomerate. Finally, since optical methods are sensitive to the square of the particle volume [i.e. $\left(D^{\text {hyd }}\right)^{6}$ ], they generally overemphasize the large-particle exponentially decreasing tail of the size distribution, thus ignoring the size range containing the majority of particles. For these reasons, it is highly desirable to have a method for performing an in-situ determination of the hydrodynamic particle diameter in a concentrated, as opposed to diluted, ferrofluid.

Recently, a new measurement technique based on the principle of the torsional pendulum (TP) [10] was introduced, which allows determining the transverse magnetic relaxation time and rotational viscosity of a ferrofluid. This method has the advantage of being suitable for investigating undiluted samples. 
Table 1. Specifications of the investigated ferrofluids. The volume fraction $\varphi$ of the ferromagnetic material is deduced from the measured saturation magnetization $M_{\mathrm{s}}$ using the relation $\varphi=M_{\mathrm{s}} / M_{\mathrm{B}}$, where $M_{\mathrm{B}}$ is the tabulated value for the bulk magnetization of the ferromagnetic material at room temperature $\left(M_{\mathrm{B}}=4.46 \times 10^{5} \mathrm{~A} / \mathrm{m}\right.$ for magnetite and $14.01 \times 10^{5} \mathrm{~A} / \mathrm{m}$ for cobalt). The quantity $\chi_{\mathrm{i}}$ is the magnetic susceptibility, as determined from the initial slope of $M v s$. $H$, and $\eta$ is the dynamic viscosity, as provided by the manufacturer.

\begin{tabular}{ccccc}
\hline Ferrofluid & $M_{\mathrm{s}}(\mathrm{A} / \mathrm{m})$ & $\varphi(\%)$ & $\chi_{\mathrm{i}}$ & $\eta(\mathrm{Pa} \mathrm{s})$ \\
\hline APG 933 & 18139. & 4.1 & 1.09 & 0.5 \\
APG 934 & 16398. & 3.7 & 0.73 & 1. \\
APG 935 & 17885. & 4.0 & 0.58 & 1.5 \\
APG 936 & 19010. & 4.3 & 0.85 & 2. \\
Co & 6512. & 1.5 & 0.44 & 0.5 \\
\hline
\end{tabular}

Furthermore, unlike other methods for recording the rotational viscosity, the flow profile during the measurement is free of disturbance by shear. By fitting the measured dissipative torque to an appropriate microscopic model, one can obtain volume-averaged estimates for both $D$ and $D^{\text {hyd }}$.

In this paper we present a comprehensive microstructural characterization of a series of ferrofluids, comparing the results obtained from TP measurements with those of several standard, complementary techniques used for colloidal structure analysis. The latter include magneto-granulometry (MG), X-ray diffraction (XRD), transmission electron microscopy (TEM), and photon-correlation spectroscopy (PCS).

\section{Sample preparation}

In these experiments four commercially available magnetite-based ferrofluids, APG 933-936 (Ferrofluidics, Nürtingen), and a sample composed of cobalt particles (Kardiotechnik, Berlin) were investigated. The magnetite grains of the APG series are coated with a tenside layer (of unknown specifications) and suspended in a hydrocarbon carrier liquid. The individual sample fluids of the APG series differ in their respective viscosities. According to the manufacturer, the magnetite volume fraction in each of the APG samples is $\varphi \simeq 3.3 \%$; however, our own measurements of the saturation magnetization (Sect. 3) reveal significant concentration differences, as indicated in Table 1. For sample characterization by TEM and by light scattering, the APG fluids were diluted with $n$-heptane to ratios as low as $1: 400$.

The Co particles were coated by lauryol-sarcosine and dispersed in a diffusion pump oil (Edwards L9). A thickener agent consisting of either polyisobutylene-succinimide or Lubrizol 7761A was added to enhance the viscosity of the sample. A dilution series (to ratios as low as $1: 2000$ ) of the Co- 
based ferrofluid, necessary for the light-scattering experiments and the TEM analysis, was prepared by adding carrier oil. From measurements of the saturation magnetization, we estimated the volume fraction of cobalt in the undiluted samples to be $\varphi=1.5 \%$.

\section{Magneto-granulometry (MG)}

The distribution $g(D)$ of ferromagnetic core diameters $D$ in a particular sample can be derived from the equilibrium magnetization curve $M_{\text {eq }}$ in an external field $H_{\text {ext }}$. In a dilute sample of monodisperse ferromagnetic colloids (i.e. one in which dipolar interactions between particles can be neglected), one expects the magnetization to follow the Langevin behavior of an ideal dipolar gas, which has the form $M_{\mathrm{eq}}=M_{\mathrm{s}} \mathcal{L}(\alpha)$, where $M_{\mathrm{s}}$ is the saturation magnetization, $\mathcal{L}(\alpha)$ denotes the Langevin function $\operatorname{coth}(\alpha)-1 / \alpha$, and $\alpha$ is the Langevin parameter $\mu_{0} m H_{\text {ext }} /\left(k_{\mathrm{B}} T\right)$, with particle magnetic moment $m=\pi D^{3} M_{\mathrm{B}} / 6$. Here, $\mu_{0}$ denotes the vacuum permeability, $M_{\mathrm{B}}$ the bulk magnetization of the magnetic material, $k_{\mathrm{B}}$ the Boltzmann constant and $T$ the temperature.

In a real ferrofluid the polydispersity of the colloid and the interparticle interactions lead to noticeable deviations from ideal Langevin behavior [11]. The polydispersity can be approximated by assuming a lognormal distribution of particle sizes:

$$
g(D)=\frac{1}{\sqrt{2 \pi} D \ln \sigma} \exp \left[-\frac{\ln ^{2}\left(D / D_{0}\right)}{2 \ln ^{2} \sigma}\right],
$$

where $D_{0}$ is the median and $\sigma$ the geometric standard deviation of the distribution. If the dipolar coupling is not too strong and the concentration $\varphi$ not too high, the dipolar particle interaction can be approximated within the mean-field Weiss model [11]. To that end the external field $H_{\text {ext }}$ appearing in the Langevin parameter $\alpha$ must be replaced with the local magnetic field $H_{\text {loc }}$ seen by an individual particle. The latter is related to the external field through $H_{\mathrm{loc}}=H+$ $(M / 3)$, where $M / 3$ is the Lorentz cavity field $[12,13], H=H_{\text {ext }}-\bar{N} M$ is the macroscopic field inside the sample, and $\bar{N} M$ is the demagnetization related to the sample geometry. For our sample geometry we have $H_{\mathrm{loc}} \simeq H_{\mathrm{ext}}-0.11 \mathrm{M}$.

The equilibrium magnetization $M_{\mathrm{eq}}$ of the ferrofluid can then be written in the following integral representation

$$
M_{\mathrm{eq}}=M_{\mathrm{s}} \frac{\int_{0}^{\infty} x^{3} g(x) \mathcal{L}\left(\mu_{0} M_{\mathrm{B}} \frac{\pi x^{3}}{6 k_{\mathrm{B}} T} H_{\mathrm{loc}}\right) \mathrm{d} x}{\int_{0}^{\infty} x^{3} g(x) \mathrm{d} x} .
$$

The parameters $D_{0}, \sigma$ and $M_{\mathrm{s}}$ are then varied to achieve the best possible fit between Eq. (2) and a given measured magnetization curve. We recorded the magnetization of the ferrofluid samples using a vibrating-sample magnetometer (Lake Shore VSM, Type 7300). The dashed line in Fig. 1 is 


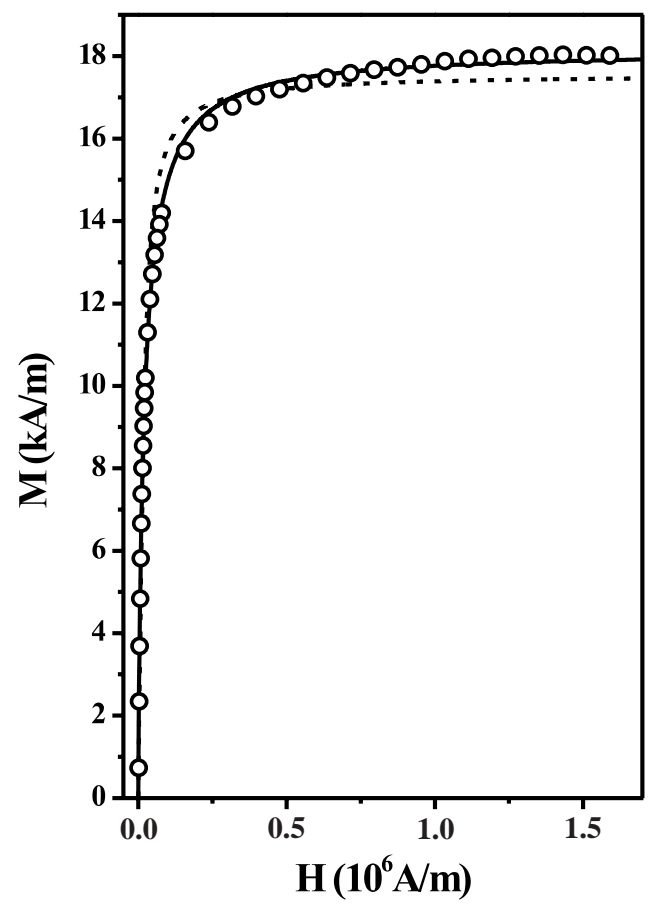

Fig. 1. Symbols denote the measured equilibrium magnetization for APG 933 as a function of the macroscopic magnetic field $H$ within the sample. The solid line is a leastsquares fit according to Eq. (2) with $D_{0}, \sigma$, and $M_{\mathrm{s}}$ as given in Table 2. The dashed line is a fit for a monodisperse ferrofluid (Langevin behavior) with $D=11.1 \mathrm{~nm}$ and $M_{\mathrm{s}}=17563 \mathrm{~A} / \mathrm{m}$.

a pure Langevin fit to such a magnetization curve, indicating that a monodisperse particle distribution $(\sigma=1)$ is a rather poor approximation for the true particle-size distribution. A lognormal distribution of particle diameters, however, results in close agreement between measured and calculated magnetization curves (Fig. 1). The lognormal distribution parameters obtained from magneto-granulometry analyses of the ferrofluid samples are listed in Table 2. Additionally, Table 2 includes values for the volume-averaged diameter $D_{\mathrm{v}}=\left\langle D^{3}\right\rangle^{1 / 3}=D_{0} \exp \left[(3 / 2) \ln ^{2} \sigma\right]$, which can be measured by the torsional-pendulum method (Sect. 7), and for the volume-weighted average diameter $D_{\text {vol }}=\left\langle D^{4}\right\rangle /\left\langle D^{3}\right\rangle=D_{0} \exp \left[(7 / 2) \ln ^{2} \sigma\right]$, which is the size parameter customarily provided by X-ray diffractometry (Sect. 4).

To rule out a possible concentration dependence of these parameters, we investigated a dilution series with $n$-heptane ranging from the non-diluted volume concentration of $\varphi \simeq 3.3 \%$ down to concentrations as low as $0.019 \%$. No systematic dependence of $D_{0}$ and $\sigma$ on concentration was found. From the scatter in the parameter values, we estimate the uncertainties associated with the 
Table 2. The core-size distribution parameters $D_{0}$ and $\sigma$ and the saturation magnetization $M_{\mathrm{s}}$ for the ferrofluid samples, as determined by magneto-granulometry using Eq. (2). Also listed are the volume-averaged core diameter $D_{\mathrm{v}}=\left\langle D^{3}\right\rangle^{1 / 3}$ and the volume-weighted core diameter $D_{\text {vol }}=\left\langle D^{4}\right\rangle /\left\langle D^{3}\right\rangle$.

\begin{tabular}{cccccc}
\hline Ferrofluid & $M_{\mathrm{s}}(\mathrm{A} / \mathrm{m})$ & $D_{0}(\mathrm{~nm})$ & $\sigma$ & $D_{\mathrm{v}}(\mathrm{nm})$ & $D_{\text {vol }}(\mathrm{nm})$ \\
\hline APG 933 & 18149. & 7.0 & 1.47 & 8.7 & 11.7 \\
APG 934 & 16400. & 7.1 & 1.42 & 8.5 & 10.9 \\
APG 935 & 17886. & 6.3 & 1.44 & 7.6 & 9.7 \\
APG 936 & 19013. & 7.0 & 1.42 & 8.4 & 10.8 \\
Co & 6512. & 11.0 & 1.27 & 12.0 & 13.5 \\
\hline
\end{tabular}

magneto-granulometric size determination to be $\pm 1.0 \mathrm{~nm}$ for $D_{0}$ and \pm 0.02 for $\sigma$.

\section{X-ray diffraction (XRD)}

Using X-ray diffraction, we determined the volume-weighted average core size of the ferrofluid particles. A glass substrate holder was filled with undiluted ferrofluid and mounted in a Siemens D-5000 diffractometer with $\theta-\theta$ geometry. Wide-angle diffraction scans were recorded from $10^{\circ}$ to $140^{\circ}(2 \theta)$ with a typical step size of $0.02^{\circ}$ and count time of $20 \mathrm{~s}$ using $\mathrm{Cu}-K_{\alpha}$ radiation $(\lambda=1.54 \AA)$. Fluorescence from Fe was suppressed by energy filtration of the secondary beam using a solid-state detector. All Bragg peaks recorded in the diffraction measurements of the ferrofluid samples APG 933-936 could be indexed to magnetite $\left(\mathrm{Fe}_{3} \mathrm{O}_{4}\right)$; no evidence for the presence of secondary phases was found. The Bragg peaks were broadened considerably with respect to the peak widths expected for diffraction from a coarse-grained $(>1 \mu \mathrm{m})$, strain-free sample of magnetite. This indicates the presence of a significant amount of inhomogeneous lattice strain and/or a broad size distribution of the magnetite cores, with an average size well below $50 \mathrm{~nm}$ [14]. By performing a standard peak-profile analysis according to the method of Warren and Averbach [15-18] or a full-pattern profile fit [19], it is possible to separate the lattice-strain effect from that of the crystallite size and thereby determine the inhomogeneous strain level $e$ and the volume-weighted average crystallite size $D_{\mathrm{vol}}$. Furthermore, one can also estimate the geometric standard deviation $\sigma$ of the lognormal distribution of spherical crystallites that would give rise to Bragg peak profiles most nearly identical to those actually measured [19].

The result of a full-pattern profile fit to the diffraction scan of sample APG 936 is shown in Fig. 2. This fitting technique simultaneously refines $D_{\text {vol }}$, $\sigma, e$, the lattice parameter of magnetite and a polynomial background function in order to obtain a least-squares fit to the measured diffraction intensity. In 


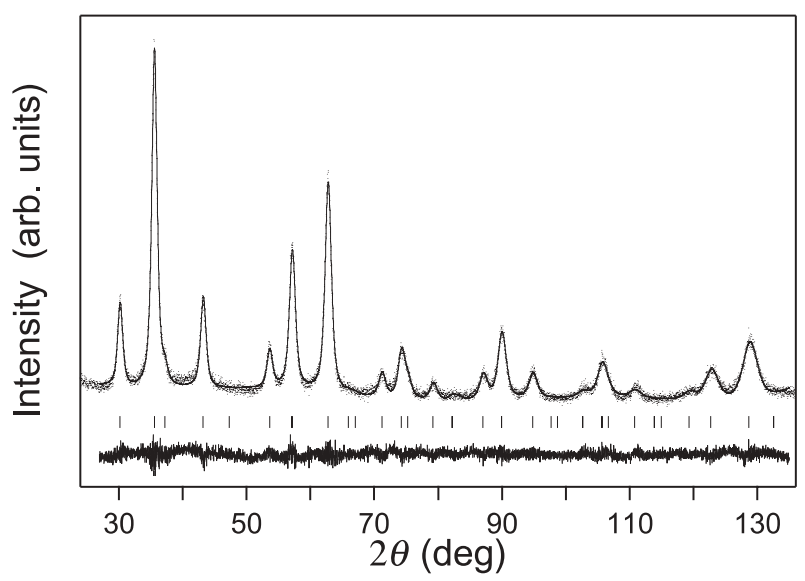

Fig. 2. X-ray diffraction scan of ferrofluid APG 936 (dots). The solid curve superimposed on the measured data is the result of a full-pattern profile fit between $27^{\circ}$ and $135^{\circ}$; below that lies the difference curve between the measured and calculated diffraction intensities. Vertical lines indicate the expected positions of Bragg reflections from $\mathrm{Fe}_{3} \mathrm{O}_{4}$. The fit curve represents the calculated diffraction intensity arising from a lognormal distribution of spherical crystallites with a median diameter of $D_{0}=5.3 \mathrm{~nm}$ and a geometric standard deviation of $\sigma=1.60$.

Table 3. The parameters $D_{0}$ and $\sigma$ of a lognormal distribution of crystallite sizes, as obtained from XRD analysis of the magnetite-based ferrofluids. Also included are the volume-averaged diameter $D_{\mathrm{v}}=\left\langle D^{3}\right\rangle^{1 / 3}$ and the volume-weighted average diameter $D_{\text {vol }}=\left\langle D^{4}\right\rangle /\left\langle D^{3}\right\rangle$.

\begin{tabular}{ccccc}
\hline Ferrofluid & $D_{0}(\mathrm{~nm})$ & $\sigma$ & $D_{\text {v }}(\mathrm{nm})$ & $D_{\text {vol }}(\mathrm{nm})$ \\
\hline APG 933 & 5.4 & 1.64 & 7.8 & 12.7 \\
APG 934 & 6.3 & 1.54 & 8.4 & 12.1 \\
APG 935 & 5.7 & 1.53 & 7.5 & 10.7 \\
APG 936 & 5.3 & 1.60 & 7.3 & 11.5 \\
\hline
\end{tabular}

Table 3 the results of full-pattern profile fits to the four APG ferrofluid specimens are collected. Within the uncertainty range of this analysis technique $( \pm 10 \%)$, the crystallite sizes and lattice strains were found to be identical for all samples. Averaging over the indicated values, we find $D_{\text {vol }}$ equal to $(11.8 \pm 0.7) \mathrm{nm}$ and $\sigma$ equal to $1.58 \pm 0.04$. Averaging over the inhomogeneous lattice-strain values yields $e=(0.29 \pm 0.05) \%$.

Comparing the size distributions obtained by XRD with those of MG, we observe that the $D_{0}$ values from XRD are systematically smaller (on average by $20 \%$ ), and the width parameter $\sigma$ is larger by about $8 \%$. However, the results of the two methods are consistent within the ranges of experimental uncertainty 
for $D_{0}$ and $\sigma$. The agreement between the values obtained by each method for the volume-averaged diameter $D_{\mathrm{v}}$ is even better, with a mean difference of less than $7 \%$.

It should be emphasized that the "crystallite size" measured by X-ray diffraction need not be identical to the size of the magnetite cores making up each particle in a ferrofluid. This is because such a core may contain two or more crystallites separated by large-angle grain boundaries. However, we could rule out this possibility for the samples APG 933-936 by the use of highresolution TEM, as outlined in the next section.

\section{Transmission electron microscopy (TEM)}

Samples for TEM analysis were prepared by diluting the magnetite-based ferrofluid samples with $n$-heptane in an ultrasonic bath and then transferring a small amount to copper grids coated with amorphous carbon. Brightfield/dark-field image pairs and electron-diffraction patterns were recorded at $200 \mathrm{kV}$ on a JEOL 2010 microscope. Cryo-TEM investigations were performed on APG 933, comparing an undiluted sample $(\varphi=4.1 \%)$ with a diluted one $(\varphi=0.007 \%)$. The cryo-TEM studies - performed by the base-grid method - entailed shock-freezing the sample holder carrying the ferrofluid before introducing it into the TEM. In addition, a high-resolution TEM analysis was carried out on individual magnetite particles in highly diluted specimens.

Unlike X-ray diffraction scans, dark-field TEM images provide a direct measure for the spatial extent of the individual crystallites in a sample (see inset in Fig. 3). From such images one can determine the crystallite-size distribution without having to rely on an assumed distribution shape, as is the case with XRD or MG. Histograms approximating the crystallite-size distribution in the ferrofluid samples were generated by computer-assisted analysis of several dark-field images (Fig. 3). The resulting distribution can indeed be closely approximated by a lognormal function, as was assumed in the XRD and MG analyses. Furthermore, the corresponding values for $D_{0}$ and $\sigma$ are very close to those obtained by XRD and MG.

To investigate the possible occurrence of particle agglomeration caused by diluting the sample with $n$-heptane or subsequent evaporation of heptane from the sample while in the microscope, we performed cryo-TEM investigations of a series of ferrofluids of varying concentration. Fig. 4 shows the images of an undiluted (a) and highly diluted (b) sample of APG 936. The associated crystallite-size histograms shown in (c) do not differ significantly, indicating that there is no measurable particle agglomeration.

Fig. 4(a) also sheds light on the hydrodynamic diameter of the particles in the APG 936 sample. Taking the undiluted volume fraction of $\varphi=4.3 \%$ and the measured particle core diameter of $D_{\mathrm{v}} \simeq 8.4 \mathrm{~nm}$ (Tables 1 and 2), one obtains for the average light distance between the surfaces of adjacent particles 


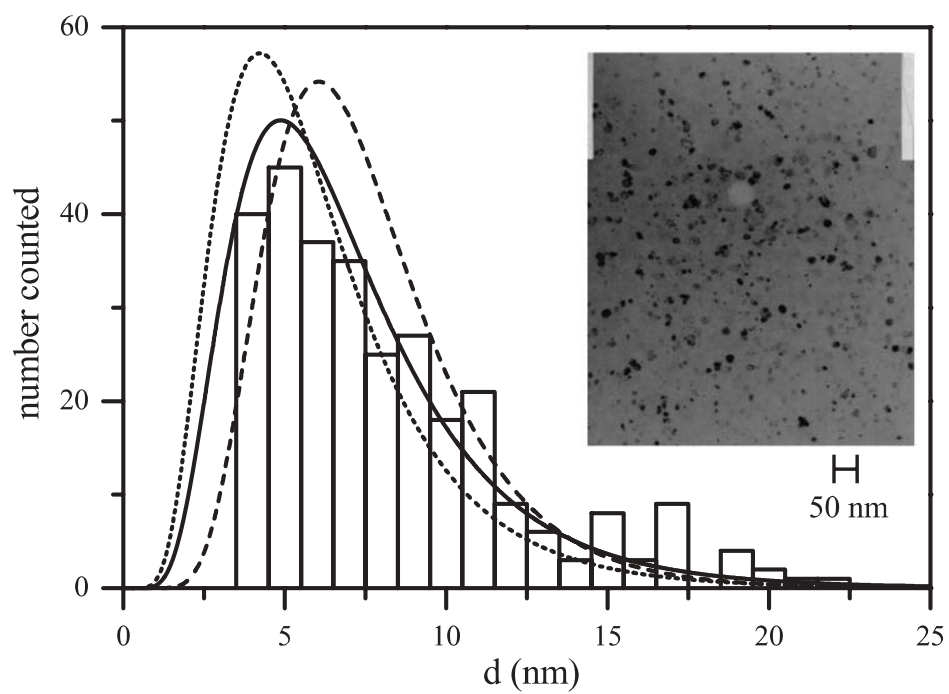

Fig. 3. Histogram of the crystallite-size distribution of APG 933, as counted from 12 darkfield TEM negatives like the one shown in the inset. The data are well represented by a lognormal fit (solid curve) with $D_{0}=6.2 \mathrm{~nm}$ and $\sigma=1.6$, giving $D_{\mathrm{v}}=7.7 \mathrm{~nm}$. The dashed curve is the size distribution obtained from MG, and the dotted curve is the result of XRD.

$11 \mathrm{~nm}$. However, the analysis of Fig. 4(a) (also performed on the undiluted sample) reveals that the particles are able to approach closer to each other. Here the evaluated average light distance is only $(5.2 \pm 1.7) \mathrm{nm}$. We take half of this value as an upper limit for the thickness of the particle coating.

The possible existence of grain boundaries in the magnetite cores was investigated by examining high-resolution TEM images of individual particles. When a magnetite core is oriented correctly relative to the electron beam, it is possible to determine whether the atomic planes of a crystallite within the core extend to the edges of the core region or terminate at an internal boundary (Fig. 5). We found no evidence in such studies for the presence of high-angle boundaries within the magnetite cores, implying that each is comprised of a single crystallite of $\mathrm{Fe}_{3} \mathrm{O}_{4}$. Thus, the crystallite sizes yielded by $\mathrm{X}$-ray diffraction and dark-field TEM analysis represent the size of the entire magnetite core.

\section{Photon-correlation spectroscopy (PCS)}

Dynamic light-scattering experiments performed on diluted ferrofluid samples yield a value for the diffusion coefficient of the particles, which in turn depends on the hydrodynamic particle diameter $D^{\text {hyd }}$. In our studies, 


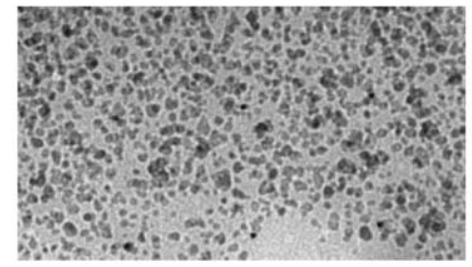

a)

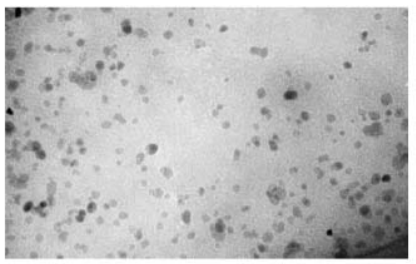

b) $100 \mathrm{~nm}$

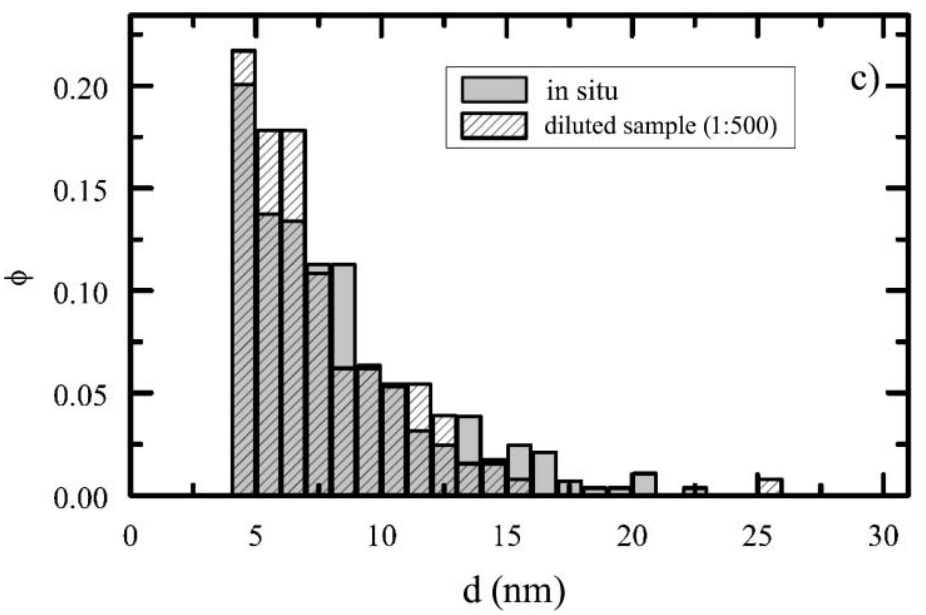

Fig. 4. Crystallite-size histograms (c) obtained by cryo-TEM studies of an undiluted sample (a) of APG 936 (grey histogram bars) and after strong dilution (b) with $n$-heptane to $\varphi=0.0067 \%$ (striped histogram bars).

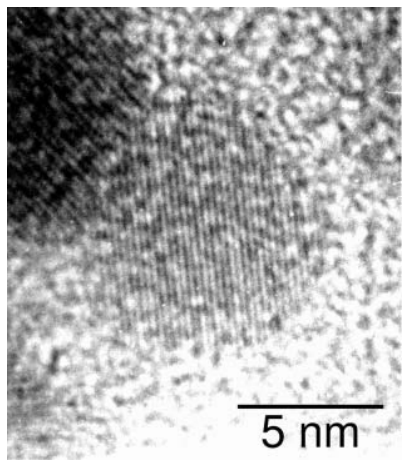

Fig. 5. High-resolution TEM image of magnetite cores in specimen APG 936. The (311) atomic planes of the ferrofluid particle in the center of the image are visible as nearly vertical lines. Since the atomic planes extend unbroken to the edges of the core, we can conclude that the particle contains no internal high-angle grain boundaries. 


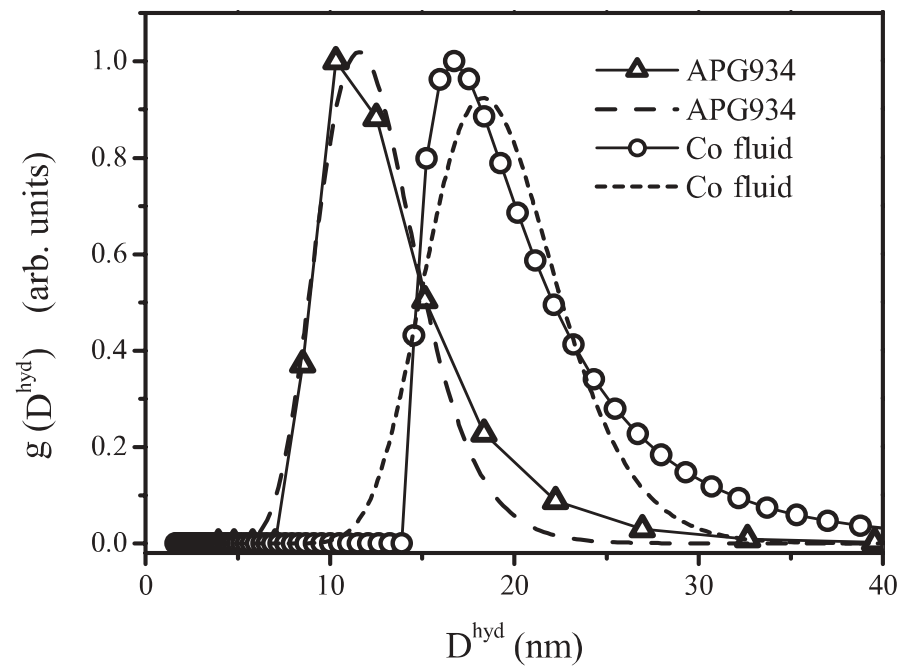

Fig. 6. Number-weighted distribution $g\left(D^{\text {hyd }}\right)$ for the hydrodynamic diameter, as obtained from the CONTIN regularization of the intensity correlation. Fitting the curves with a lognormal distribution (dashed lines) yields the parameters $D_{0}$ and $\sigma$ listed in Table 4.

photon-correlation spectroscopy was carried out on an ALV 5000 spectrometer equipped with a krypton-ion laser (Spectra Physics). By varying the laser power between 8 and $30 \mathrm{~mW}$, we could rule out the influence of absorptive heating. Measurements were made at scattering angles between $60^{\circ}$ and $150^{\circ}$ to confirm that the intensity correlation results from translational diffusion of the colloid particles. Moreover, by testing different scattering angles, one obtains an estimate for the experimental uncertainties in the value for the diffusion coefficient. Dilution series were investigated with volume fractions ranging from $\varphi=0.002 \%$ to $0.02 \%$. To eliminate dust or other larger particles, the samples were filtered by a membrane filter with a pore size of $200 \mathrm{~nm}$. The intensity correlation function of the scattered light was recorded with an ALV-5000E correlator. Thereafter, the data were fitted using the ALV-NonLin routine, which is based on the CONTIN regularization [20]. This analysis yields an intensity-weighted distribution function for the decay times. Under the assumption that the scattering particles behave like hard spheres in dilute solution, one can deduce the distribution of the hydrodynamic radii from the Stokes-Einstein relation. Applying an appropriate data weighting, one then obtains the number-weighted hydrodynamic-diameter distribution function $g\left(D^{\text {hyd }}\right)$ from the intensityweighted distribution.

Fig. 6 illustrates the distribution $g\left(D^{\text {hyd }}\right)$ for the magnetite-based ferrofluid APG 934 and for the Co-based sample. By fitting such CONTIN curves with a lognormal distribution (dashed lines), we obtain the size parameters $D_{0}$ 
Table 4. The parameters $D_{0}$ and $\sigma$ of a lognormal distribution of hydrodynamic diameters, as obtained from PCS measurements. Values for the volume-averaged hydrodynamic diameter $D_{\mathrm{v}}=\left\langle D^{3}\right\rangle^{1 / 3}$ and the volume-weighted average hydrodynamic diameter $D_{\text {vol }}=\left\langle D^{4}\right\rangle /\left\langle D^{3}\right\rangle$ are also listed.

\begin{tabular}{cllll}
\hline Ferrofluid & $D_{0}(\mathrm{~nm})$ & $\sigma$ & $D_{\mathrm{v}}^{\text {hyd }}(\mathrm{nm})$ & $D_{\text {vol }}^{\text {hyd }}(\mathrm{nm})$ \\
\hline APG 934 & 12.1 & 1.2 & 12.7 & 14. \\
APG 935 & 14.5 & 1.2 & 15.2 & 16.8 \\
APG 936 & 18.6 & 1.2 & 19.5 & 21.6 \\
Co & 19. & 1.2 & 20. & 22. \\
\hline
\end{tabular}

and $\sigma$, which can be compared directly to the results of the other sample characterizations. The resulting size parameters are listed in Table 4 together with the mean values $D_{\mathrm{v}}^{\text {hyd }}$ and $D_{\mathrm{vol}}^{\text {hyd }}$. From the scatter in measurements made at different angles, we estimate the uncertainty of the mean particle size to be $\pm 1.5 \mathrm{~nm}$.

\section{Torsional pendulum (TP)}

As first shown by McTague [2], the effective viscosity of a ferrofluid is enhanced in a static magnetic field $\mathbf{H}[1,3]$. The origin of this extra dissipation is the slow relaxation of the magnetization, which leads to a perceptible deviation between the actual magnetization $\mathbf{M}(\mathbf{r}, t)$ and its equilibrium value $\mathbf{M}_{\mathrm{eq}}(\mathbf{r}, t)$, resulting in a torque density $\mu_{0}(\mathbf{M} \times \mathbf{H})$. Microscopically, the magneto-viscous effect can be explained by the enhanced viscous friction experienced by a particle in a rotational flow field when the orientation of the particle is held fixed by an external magnetic field.

The magneto-dissipative energy loss can be measured by a torsional pendulum (TP) with a high quality factor that is forced to execute small harmonic torsional oscillations described by $\ddot{\phi}+\Gamma \dot{\phi}+\omega_{0}^{2} \phi=F \cos \omega t$ [10]. Here $\phi$ denotes the deflection angle, $\Gamma$ the damping increment, $\omega_{0}$ the eigenfrequency of the oscillator, and $F$ the strength of the external drive. The enhancement of $\Gamma$ in a static external field is determined by recording the resonance curve of the TP. In order to avoid disturbance from shear flow within the sample and to guarantee rigid rotation of the fluid with vorticity $\Omega(t)=\dot{\phi}(t) \propto \cos (\omega t)$, the ferrofluid volume is partitioned into a large number of subvolumes. The absence of a noticeable parasitic shear flow was checked by a control experiment with the ferrofluid replaced by a solid payload. Thereby we measured at $H=0$ the same dissipation independent of whether the solid or the fluid payload was carried. Details of such an apparatus are presented elsewhere [10]. Damping rates $\Gamma(H)$ measured in two different ferrofluids using a TP are plotted in Fig. 7. 


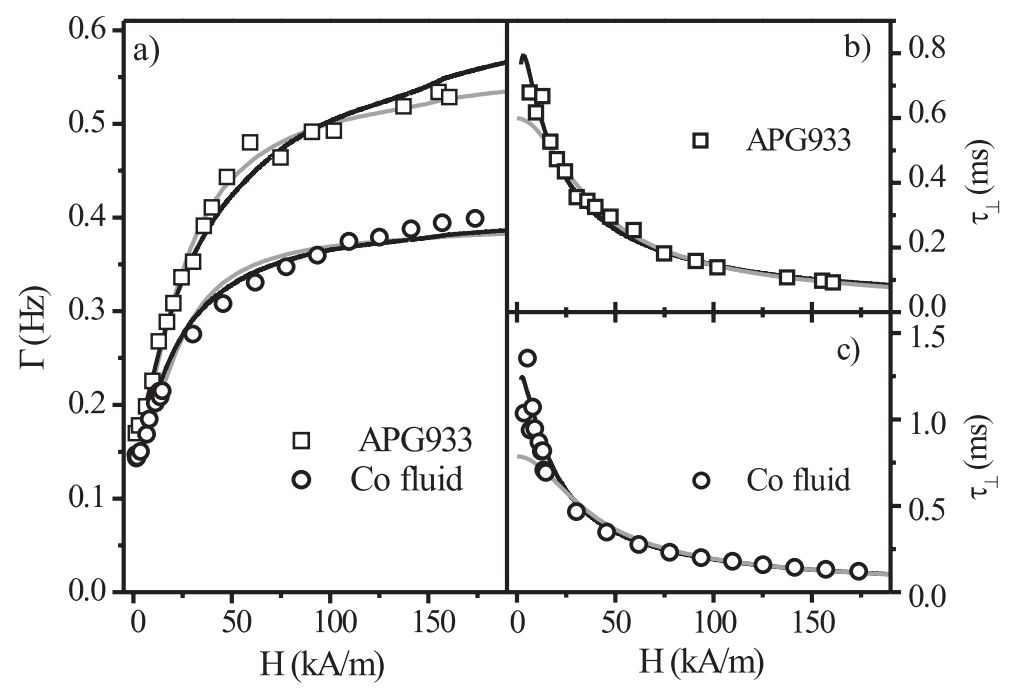

Fig. 7. (a) Symbols denote experimentally measured damping rates $\Gamma(H)$ of the TP. Black curves are fits of Eq. (7), with $\bar{\zeta}$ as listed in Table 5. Grey curves are fits of Eq. (4), with $\tau_{\perp}(H)$ given by Eq. (8) and $D_{\mathrm{v}}$ and $\tau_{\mathrm{B}}$ as listed in Table 6. Symbols in (b) and (c) denote the measured transverse relaxation time. The black and grey curves are fits according to Eqs. (6) and (8), respectively.

Since the torsional pendulum is operated at a very small deflection amplitude $\phi_{\max }$, the magnetization $\mathbf{M}$ deviates only slightly from the static equilibrium value $\mathbf{M}_{\mathrm{eq}}=M_{\mathrm{eq}} \mathbf{H} / H$. The dynamics of the off-equilibrium component $\mathbf{m}=\mathbf{M}-\mathbf{M}_{\mathrm{eq}}$ are governed by the relaxation equation

$$
\partial_{t} \mathbf{M}-\boldsymbol{\Omega} \times \mathbf{M}=-\frac{1}{\tau_{\perp}} \mathbf{m}_{\perp}-\frac{1}{\tau_{\|}} \mathbf{m}_{\|} .
$$

Here, the indices $\|$ and $\perp$ refer to the respective directions relative to the applied magnetic field. As outlined in Ref. [10], the total damping rate,

$$
\Gamma(H)=\Gamma_{\mathrm{P}}+\Gamma_{\mathrm{FF}}=\Gamma_{\mathrm{P}}+\mu_{0} \frac{V}{\Theta} H M_{\mathrm{eq}}(H) \tau_{\perp}(H),
$$

of the TP is made up of the parasitic contribution $\Gamma_{\mathrm{P}}-$ arising from air friction plus other losses - and the field-dependent magneto-viscous part, where $V$ is the volume of the sample and $\Theta$ the total moment of inertia of the TP. We note that the torsional pendulum allows a direct measurement of the socalled rotational viscosity $\eta_{\mathrm{R}}[3]$. The latter can be identified by equating the magneto-viscous force density $\frac{\mu_{0}}{2} \nabla \times(\mathbf{M} \times \mathbf{H})$ to an effective viscous force 
Table 5. The parasitic damping increment $\Gamma_{\mathrm{P}}$ and the relaxation parameter $\bar{\zeta}$, as obtained by fitting Eq. (7) to the measured $\Gamma(H)$ data.

\begin{tabular}{ccc}
\hline Ferrofluid & $\Gamma_{\mathrm{P}}(\mathrm{Hz})$ & $\bar{\zeta}(\mathrm{kHz})$ \\
\hline APG 933 & 0.17 & 1.00 \\
APG 934 & 0.14 & 0.77 \\
APG 935 & 0.13 & 0.96 \\
APG 936 & 0.17 & 0.71 \\
Co & 0.14 & 0.31 \\
\hline
\end{tabular}

density $\eta_{\mathrm{R}} \nabla^{2} \mathbf{v}$, yielding [10]

$$
\eta_{\mathrm{R}}=\frac{\Theta}{4 V} \Gamma_{\mathrm{FF}}
$$

Knowing the equilibrium magnetization curve $M_{\text {eq }}(H)$, we can also deduce the field dependence of the transverse relaxation time $\tau_{\perp}(H)$ from a measurement of $\Gamma(H)$. This result can be used to determine a nonequilibrium transport coefficient as follows. In Ref. [21] the general structure of the ferro-hydrodynamic evolution equations, including the relaxation equation for $\mathbf{M}$, was developed. For small deviations of the magnetization from local equilibrium, this theory relates $\tau_{\perp}(H)$ to a transport coefficient $\zeta(H)$ via

$$
\tau_{\perp}(H)=\frac{M_{\mathrm{eq}}(H)}{H \zeta(H)} .
$$

Within the framework of the macroscopic theory [21], the field dependence of $\zeta$ is not closer specified. However, if Eq. (6) is substituted into Eq. (4), we find that the experimental values for $\Gamma(H)$ can well be fit with a constant, $H$-independent transport coefficient $\zeta(H)=\bar{\zeta}$. Thus, the fit formula

$$
\Gamma(H)=\Gamma_{\mathrm{P}}+\mu_{0} \frac{V}{\Theta} \frac{M_{\mathrm{eq}}^{2}(H)}{\bar{\zeta}}
$$

yields values for $\Gamma_{\mathrm{P}}$ and for the transport coefficient $\bar{\zeta}$ from experimental data for $\Gamma(H)$. Fit curves generated using Eq. (7) are shown as black lines in Fig. 2(a), and the best-fit parameter values for $\Gamma_{\mathrm{P}}$ and $\bar{\zeta}$ are collected in Table 5. Inserting $\bar{\zeta}$ from Table 5 into Eq. (6) yields a simple expression for the magnetic-field dependence of the transverse relaxation time $\tau_{\perp}(H)$. In Fig. 7(b) and (c) we compare this prediction with experimental data for $\tau_{\perp}(H)$.

We now interpret the damping measurement in terms of a mesoscopic model linking the particle-size characteristics to an expression for the relaxation time $\tau_{\perp}(H)$, the parameters of which $-D$ and $D^{\text {hyd }}-$ can be optimized to achieve agreement with experimental data. Within this approach an expression 
of the form

$$
\tau_{\perp}(H)=2 \tau_{\mathrm{B}} \mathcal{L}(\alpha) /[\alpha-\mathcal{L}(\alpha)]
$$

for the transverse relaxation time has been derived [22, 23]. Here, $\tau_{\mathrm{B}}$ denotes the Brownian relaxation time, $\mathcal{L}(\alpha)=\operatorname{coth} \alpha-1 / \alpha$ the Langevin function, and $\alpha=\mu_{0} m H / k_{\mathrm{B}} T$ the Langevin parameter. The latter is proportional to the magnetic moment $m$ of the ferrofluid particles and thus to the core volume $\pi D_{\mathrm{v}}^{3} / 6$. To arrive at Eq. (8), the following approximations were made: (i) the suspended particles are monodisperse, (ii) they have a spherical hydrodynamical shape, (iii) they are non-interacting, and (iv) the magnetic moment of each particle is fixed in orientation relative to the corresponding magnetic core (rigid-dipole approximation). The particles undergo Brownian rotational diffusion in the carrier fluid and experience magnetic torques in the presence of a magnetic field.

It is clear that the approximations listed above imply idealizations of real ferrofluid properties. First, it has been demonstrated in Sect. 3 that the assumption of a monodisperse particle-size distribution is rather crude. Therefore, such a microscopic interpretation can provide access only to the volumeaveraged values $D_{\mathrm{v}}$ and $D_{\mathrm{v}}^{\text {hyd }}$ but not to their distributions. Second, by means of the TEM analysis in Sect. 5, we found that the ferromagnetic cores are only approximately spherical. However, with the surfactant coating taken into account (possibly swollen in the carrier liquid), the hydrodynamic shell is certainly closer to spherical than the core it encloses. Third, at the investigated volume fraction of $\varphi \simeq 3.3 \%$, the magnetic susceptibility $\chi$ is of the order of unity, and the neglect of dipolar interactions is inappropriate due to a considerable demagnetization effect. However, as outlined in Sect. 3, this problem can largely be remedied at a mean-field level by replacing the external magnetic field $H_{\text {ext }}$ with the local field $H_{\text {loc }}$. Furthermore, at a typical concentration of $\varphi=3-4 \%$, the average surface-to-surface distance between ferromagnetic cores amounts to $11-12 \mathrm{~nm}$. Since the thickness of the steric coating is only a few nanometers (see below and Sect. 5), it is a reasonable approximation to disregard the hydrodynamic interaction between particles. Finally, the assumption that the ferromagnetic grains can be approximated as Brownian rigid dipoles requires justification. Shliomis [24] pointed out that magnetization relaxation in cobalt-based and iron-based ferrocolloids occurs predominantly by Brownian rotation when the particle core diameters exceed 4 or $8.5 \mathrm{~nm}$, respectively. These thresholds are in fact even somewhat lower if the form anisotropy of the particles is taken into account. The MG analysis of Sect. 3 yields $D_{\mathrm{v}} \simeq 8.0 \mathrm{~nm}$ for the cobalt-based ferrofluid and $D_{\mathrm{v}} \simeq 8.3 \mathrm{~nm}$ for the APG series. This indicates that the cobalt particles certainly behave like rigid dipoles, whereas a significant fraction of the magnetite grains may be hydrodynamically ineffective. As a conservative estimate we suppose that the ineffective fraction of magnetite grains is $50 \%$. In this case the hydrodynamic diameters $D_{\mathrm{v}}^{\text {hyd }}$ given below for the APG series are underestimated by $20 \%$. 
Table 6. Volume-averaged core diameter $D_{\mathrm{v}}$, Brownian relaxation time $\tau_{\mathrm{B}}$ and volumeaveraged hydrodynamic diameter $D_{\mathrm{v}}^{\text {hyd }}$, as obtained from the measured field dependence of the transverse relaxation time $\tau_{\perp}(H)$ using Eq. (8).

\begin{tabular}{cccc}
\hline Ferrofluid & $D_{\mathrm{v}}(\mathrm{nm})$ & $\tau_{\mathrm{B}}(\mathrm{ms})$ & $D_{\mathrm{v}}^{\mathrm{hyd}}(\mathrm{nm})$ \\
\hline APG 933 & 10.4 & 0.599 & 14.6 \\
APG 934 & 9.1 & 0.485 & 10.8 \\
APG 935 & 8.7 & 0.355 & 8.5 \\
APG 936 & 8.2 & 0.480 & 8.5 \\
Co & 7.0 & 0.786 & 16.2 \\
\hline
\end{tabular}

Using $D_{\mathrm{v}}$ and $\tau_{\mathrm{B}}$ as fit parameters in Eq. (8), we calculate the grey curves in Fig. 7(b) and (c). With the given viscosity $\eta$ of the ferrofluid, the volumeaveraged hydrodynamic particle diameter $D_{\mathrm{v}}^{\text {hyd }}$ can be obtained from the Brownian relaxation time $\tau_{\mathrm{B}}$ by virtue of the relation $\tau_{\mathrm{B}}=\eta \pi\left(D_{\mathrm{v}}^{\text {hyd }}\right)^{3} /\left(2 k_{\mathrm{B}} T\right)$. The corresponding results for the various ferrofluid samples are compiled in Table 6. The volume-averaged core diameters $D_{\mathrm{v}}$ are in good agreement with the values obtained by the MG method. Moreover, comparing $D_{\mathrm{v}}$ and $D_{\mathrm{v}}^{\text {hyd }}$, we estimate a thickness between 0.2 and $4.6 \mathrm{~nm}$ for the surfactant coating, with an average value of about $2 \mathrm{~nm}$.

\section{Discussion}

We used a torsional pendulum (TP) to measure the rotational viscosity of ferrocolloids in a shear-flow-free manner [10]. A major advantage of this method is that the measurement can be performed in situ, i.e. without diluting the sample, thus eliminating the risk of a dilution-induced change in the ferrofluid microstructure, such as particle agglomeration or swelling of the surfactant coating. Measuring the damping rate $\Gamma(H)$ of the pendulum allows a determination of the magnetic-field dependence of the transverse magnetic relaxation time $\tau_{\perp}(H)$. The measured dependence is well represented by a simple analytical expression in terms of the equilibrium magnetization curve $M_{\text {eq }}(H)$. This functional form was suggested by the general structure of the ferrohydrodynamic-evolution equations derived from underlying symmetries and conservation laws via nonequilibrium thermodynamics. Information regarding the ferrofluid microstructure was obtained within a microscopic model linking $\tau_{\perp}(H)$ to the volume-averaged core diameter $D_{\mathrm{v}}$ and to the hydrodynamic diameter $D_{\mathrm{v}}^{\text {hyd }}$ (core plus surfactant coating) of the suspended particles. The present paper compares the microstructural results obtained by the TP method with those of other standard methods, as summarized in Table 7.

With regard to the core diameter $D_{\mathrm{v}}$, we find that the TP results lie in broad agreement with those of the complementary methods [magneto-granulometry 
Table 7. Comparison of the volume-averaged core diameters $D_{\mathrm{v}}$ and the hydrodynamic diameters $D_{\mathrm{v}}^{\text {hyd }}$ yielded by the various methods employed in this study. Experimental uncertainties for each method are given in the column headings.

\begin{tabular}{|c|c|c|c|c|c|c|c|}
\hline \multirow[b]{2}{*}{$\begin{array}{l}\text { Ferrofluid } \\
\text { error } \pm(\mathrm{nm})\end{array}$} & \multicolumn{4}{|c|}{$D_{\mathrm{v}}(\mathrm{nm})$} & \multicolumn{3}{|c|}{$D_{\mathrm{v}}^{\text {hyd }}(\mathrm{nm})$} \\
\hline & $\begin{array}{c}\mathrm{MG} \\
1\end{array}$ & $\begin{array}{c}\mathrm{XRD} \\
1\end{array}$ & $\begin{array}{c}\text { TEM } \\
0.5\end{array}$ & $\begin{array}{l}\text { TP } \\
0.6\end{array}$ & $\begin{array}{c}\text { PCS } \\
1.5\end{array}$ & $\begin{array}{l}\text { TP } \\
0.6\end{array}$ & $\begin{array}{c}\text { cryo-TEM } \\
0.5\end{array}$ \\
\hline APG 933 & 8.7 & 7.8 & - & 10.4 & - & 14.6 & - \\
\hline APG 934 & 8.5 & 8.4 & - & 9.1 & 12.7 & 10.8 & - \\
\hline APG 935 & 7.6 & 7.5 & - & 8.7 & 15.2 & 8.5 & - \\
\hline APG 936 & 8.4 & 7.0 & 7.7 & 8.2 & 19.5 & 8.5 & $<10.3$ \\
\hline Co & 8.0 & - & - & 7.0 & 20. & 16.2 & - \\
\hline
\end{tabular}

(MG), X-ray diffraction (XRD) and transmission electron microscopy (TEM)]. On the other hand, the agreement between the various values for the hydrodynamic diameter $D_{\mathrm{v}}^{\text {hyd }}$ is less satisfactory. The results obtained by photoncorrelation spectroscopy (PCS) are systematically larger than those obtained by the TP method, with deviations between $12 \%$ and $130 \%$. For the magnetitebased APG fluids, part of this discrepancy may be explained by the fact that a considerable fraction of the particles are too small to behave like rigid dipoles. But even if the hydrodynamically ineffective particles amount to $50 \%$ of the total concentration, the resulting increase in the values for $D_{\mathrm{v}}^{\text {hyd }}$ as determined by the TP method would be insufficient to explain the large difference between the PCS and TP results. Moreover, the PCS measurements performed on the cobalt sample, in which the particles are definitely rigid dipoles, also tend to overestimate (albeit less strongly) the hydrodynamic particle size.

We believe that the systematic elevation of PCS size values in comparison to the TP results is connected to the dilution of the ferrofluid samples that was necessary in order to carry out the PCS measurements. As noted in Sect. 1, unusually large hydrodynamic radii have also been observed by transient optical birefringence [9], with the ratio between $D_{\mathrm{v}}^{\text {hyd }}$ and $D_{\mathrm{v}}$ reaching values as high as 10 . Clearly, such $D_{\mathrm{v}}^{\text {hyd }}$ sizes are unrealistic for concentrated ferrofluids with a volume fraction of a few percent, since the hydrodynamic shells of the particles begin to overlap when $D_{\mathrm{v}}^{\text {hyd }} / D_{\mathrm{v}}>2.5$. One might argue - owing to the enhanced sensitivity of optical methods to large particles that the increase in hydrodynamic radius results from a small number of particle agglomerates created during the dilution process. But in our experiments this possibility was ruled out by the TEM analysis, which found no indication for dilution-induced agglomeration of the ferromagnetic grains. Moreover, the cryo-TEM investigations of the concentrated samples revealed that the layer thickness of the steric particle coating did not exceed $\simeq 3 \mathrm{~nm}$. For the hydrodynamic diameter of the APG 936 sample, this implies an estimated 
$D_{\mathrm{v}}^{\text {hyd }}<10.3 \mathrm{~nm}$ (last column in Table 7), which confirms the outcome of the TP method and contradicts the PCS result. We therefore conclude that the large hydrodynamic radii obtained by optical methods probably result from the fact that PCS significantly probes the tail of the distribution of the particles and overestimates the larger particles or agglomerates present in the dispersions under investigation.

\section{Acknowledgement}

We thank N. Buske (Kardiotechnik, Berlin) for providing the cobalt-based ferrofluid, H. Bünsch (Lubrizol, Hamburg) for supplying the viscosity modifier, R. Haberkorn for providing the full-pattern profile fitting program [19], M. Schuler for performing the TEM study and L. Edelmann for carrying out the cryo-TEM investigation. This research is supported by the Deutsche Forschungsgemeinschaft (SFB 277).

\section{References}

1. For an introduction see: R. E. Rosensweig, Ferrohydrodynamics. Cambridge University Press, Cambridge, England (1985).

2. J. P. McTague, J. Chem. Phys. 51 (1969) 133.

3. M. I. Shliomis, Sov. Phys. JETP 34 (1972) 1291.

4. M. I. Shliomis and K. I. Morozov, Phys. Fluids 6 (1994) 2855.

5. J. C. Bacri, R. Perzynski, M. I. Shliomis, and G. I. Burde, Phys. Rev. Lett. 75 (1995) 2128.

6. A. Zeuner, R. Richter, and I. Rehberg, Phys. Rev. E 58 (1998) 6287.

7. F. Gazeau, B. M. Heegaard, J. C. Bacri, A. Cebers, and R. Perzynski, Europhys. Lett. 35 (1996) 609; F. Gazeau, C. Baravian, J. C. Bacri, R. Perzynski, and M. I. Shliomis, Phys. Rev. E 56 (1997) 614.

8. J. Bläsing, G. Strassburger, and D. Eberbeck, Phys. Stat. Sol. (a) 146 (1994) 595.

9. J. C. Bacri, R. Perzynski, D. Salin, and J. Servais, J. Phys. 48 (1987) 1385 and references cited therein.

10. J. P. Embs, H. W. Müller, C. Wagner, K. Knorr, and M. Lücke, Phys. Rev. E 61 (2000) R2196.

11. B. Huke and M. Lücke, Phys. Rev. E 62 (2000) 6875.

12. J. D. Jackson, Klassische Elektrodynamik. de Gruyter, Berlin (1982), Chapt. 4.

13. T. Weser and K. Stierstadt, Z. Phys. B 59 (1985) 257.

14. H. P. Klug and L. E. Alexander, X-Ray Diffraction Procedures for Polycrystalline and Amorphous Materials, 2nd ed. Wiley, New York (1974), Chapt. 9.

15. B. E. Warren and B. L. Averbach, J. Appl. Phys. 21 (1950) 595; 23 (1952) 497.

16. B. E. Warren, X-Ray Diffraction. Dover, New York (1990), Chapt. 13.

17. C. E. Krill and R. Birringer, Philos. Mag. A 77 (1998) 621.

18. H. Natter, M. Schmelzer, M. S. Löffler, C. E. Krill, A. Fitch, and R. Hempelmann, J. Phys. Chem. B 104 (2000) 2467.

19. C. E. Krill, R. Haberkorn, and R. Birringer, in Handbook of Nanostructured Materials and Nanotechnology, Vol. 2: Spectroscopy and Theory, H. S. Nalwa (Ed.). Academic Press, London (2000), Chapt. 3.

20. S. J. Provencher, Comp. Phys. Comm. 27 (1982) 213; 27 (1982) 229. 
21. H. W. Müller and M. Liu, Phys. Rev. E 64 (2001) 061405.

22. M. A. Martsenyuk, Y. L. Raikher, and M. I. Shliomis, Sov. Phys. JETP 38 (1974) 413.

23. J. T. Waldron, Y. P. Kalmykov, and W. T. Coffey, Phys. Rev. E 49 (1994) 3976.

24. M. Shliomis, Sov. Phys.-Usp. 17 (1974) 153. 